\title{
Large-strain time-temperature equivalence in high density polyethylene for prediction of extreme deformation and damage
}

\author{
J. Furmanski, E.N. Brown, B. Clements, C.M. Cady, and G.T. Gray III \\ Los Alamos National Laboratory, Los Alamos, NM, USA
}

\begin{abstract}
Time-temperature equivalence is a widely recognized property of many time-dependent material systems, where there is a clear predictive link relating the deformation response at a nominal temperature and a high strain-rate to an equivalent response at a depressed temperature and nominal strain-rate. It has been found that high-density polyethylene (HDPE) obeys a linear empirical formulation relating test temperature and strain-rate. This observation was extended to continuous stress-strain curves, such that material response measured in a load frame at large strains and low strain-rates (at depressed temperatures) could be translated into a temperature-dependent response at high strain-rates and validated against Taylor impact results. Timetemperature equivalence was used in conjuction with jump-rate compression tests to investigate isothermal response at high strain-rate while exluding adiabatic heating. The validated constitutive response was then applied to the analysis of DynamicTensile-Extrusion of HDPE, a tensile analog to Taylor impact developed at LANL. The Dyn-Ten-Ext test results and FEA found that HDPE deformed smoothly after exiting the die, and after substantial drawing appeared to undergo a pressure-dependent shear damage mechanism at intermediate velocities, while it fragmented at high velocities. Dynamic-Tensile-Extrusion, properly coupled with a validated constitutive model, can successfully probe extreme tensile deformation and damage of polymers.
\end{abstract}

\section{Introduction}

Few experimental approaches are available for unambiguously assessing the deformation response of materials under extreme mechanical loading conditions, i.e., simultaneous large strains $(\varepsilon>1)$ and high strain-rates $(d \varepsilon / d t>$ $10^{4} \mathrm{~s}^{-1}$ ) [1], see figure 1 . Servohydraulic load frames can achieve large uniform strains, but are generally limited to strain-rates less than $10 \mathrm{~s}^{-1}$ in compression, and even less in tension. The high strain-rate response of polymers has been probed above $10^{3} \mathrm{~s}^{-1}$ using a Split Hopkinson Pressure Bar (SHPB) which imposes a one-dimensional stress wave on a specimen, but is limited in strain to approximately $0.05<\varepsilon<0.3$.

While a wide range of researchers have employed the SHPB to study the high strain-rate response of polymers in compression [2-11], the high strain-rate polymer tensile response employing tensile-SHPB has been less widely studied and the data is typically limited to slightly lower strain rates to account for the longer gage length and typically does not extend to large enough strain to capture the failure response [2,9-12]. Results including SHPB testing of polymers have extended temperature-strainrate relations beyond small-strains up to approximately $\varepsilon=20 \%$ [2], providing a substantial improvement of our understanding of their viscoelastic and plastic flow response. Nevertheless, there remain limitations to achieving extreme mechanical conditions on a specimen in a uniform and easily interpretable manner.

\subsection{Time (strain-rate)-temperature equivalence}

Time-temperature equivalence is a widely recognized property of many time-dependent material systems, where there is a clear predictive link relating the deformation response at a nominal temperature and a high strain-rate to an equivalent response (i.e., stiffness or flow stress) at a depressed temperature and nominal strain-rate. This is often attributed to the deformation response being governed by a local activation process which is a function of the temperature, the applied stress, and the activation energy for the particular activated process, due to Eyring [13,14]. There have been numerous modeling attempts to capture time-temperature equivalence in polymers, and perhaps the most well-known is the Williams-Landel-Ferry (WLF) formula, which has been extremely successful for predicting small-strain deformations for a moderate window of temperatures in a variety of amorphous polymers [15].

Recent work has investigated time-temperature equivalence in a number of polymers substantially beyond the small-strain regime. Siviour and coworkers examined uniaxial deformations at a wide range of temperatures and strain-rates in polycarbonate, and polyvinyl-difluoride (PVDF), and proposed an empirical linear formula for relating these that agreed well with the data (figure 2) $[4,6]$

$$
T_{E q}=T_{\text {Test }}+A\left[\log _{10}\left(\dot{\varepsilon}_{E q}\right)-\log _{10}\left(\dot{\varepsilon}_{\text {Test }}\right)\right]
$$

This model has a single free parameter $A$, relating the temperature and strain-rate sensitivities of the stress state. Results comparing measurements of the yield strength showed that with this single free parameter in the model virtually all experimentally accessible states in temperature and strain-rate collapsed to a single trend in a given polymer. The agreement was even more remarkable when the test matrix spanned a glass transition, and an inflection was observed in the yield strength temperature dependence. The model nevertheless fit the data at temperatures both above and below the glass transition with a single value for $A$.

Jordan et al. [8] examined temperature-strain-rate equivalence in PTFE and reported similar agreement with the formula employed by Siviour. In a study by Brown 


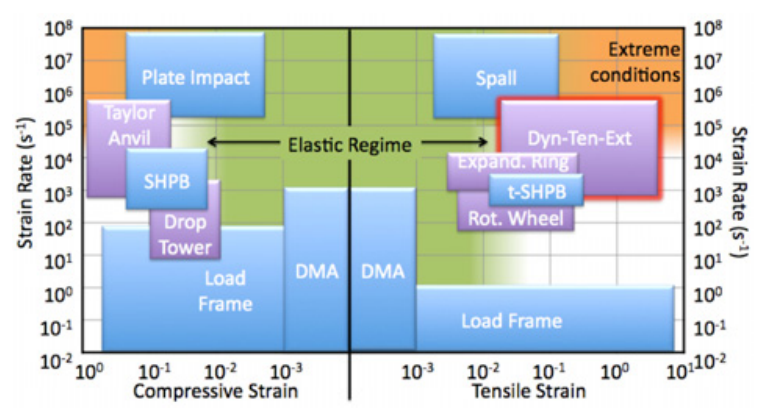

Fig. 1. Schematic of material characterization techniques over the space of strains and strain-rates.

et al., the finite strain temperature-strain-rate equivalence behavior of high density polyethylene (HDPE), ultrahigh molecular weight polyethylene (UHMWPE), and cross linked polyethylene (PEX) were studied and found to agree considerably with one another. It was also found that the temperature dependence of the yield strength exhibited no inflection over a large range of temperatures, in contrast to other polymers so investigated [7]. Their work reported the temperature-strain-rate equivalence of the yield strength, as defined as the onset of plasticity, which was observed to occur at $7.5 \%$ strain. An important finding of their work showed that one decade of increased strain-rate in these HDPE and UHMWPE formulations was equivalent to a $10.2^{\circ} \mathrm{C}$ and $10.6^{\circ} \mathrm{C}$ reduction in temperature, respectively. It is also important to note that their results also revealed substantial adiabatic heating and softening of the materials at strain-rates above $0.1 \mathrm{~s}^{-1}$. This work also tracked the temperature-strain-rate equivalence of the flow stress at $20 \%$ strain, $\sigma_{20 \%}$, corresponding to the onset of fullydeveloped plasticity, for a wide range of temperatures and applied true strain-rates. The deformation response for HDPE at these conditions (figure 2(a)-(b)), can be represented directly as the temperature and strain-rate dependence of $\sigma_{20 \%}$ can be represented (figure 2(c)-(d)) and they collapse to one equivalent response when Eq. (1) is applied with $\mathrm{A}=10.2^{\circ} \mathrm{C} /$ decade (figure 2(e)).

Special attention is paid to $\sigma_{20 \%}$ here because the deformation beyond $15 \%$ strain in monotonic loading is apparently pure plastic deformation, as the recoverable viscoelastic deformation is exhausted at that point and does not contribute to further deformation [16]. At intermediate strains where both recoverable and plastic deformation are active, the two mechanisms are confounded and could have distinct activation energies and time-temperature equivalence behaviors.

\subsection{Extreme mechanical deformations}

There has been some success in imposing extreme mechanical conditions in a controlled manner, albeit by relaxing the requirement of uniform specimen deformation. The most prominent effort in this area was first conducted by G. I. Taylor for the study of the high-rate flow stress of steel [17] through a sudden deceleration via impact of a rapidly moving specimen against a massive anvil. During these Taylor cylinder impact experiments extreme plastic

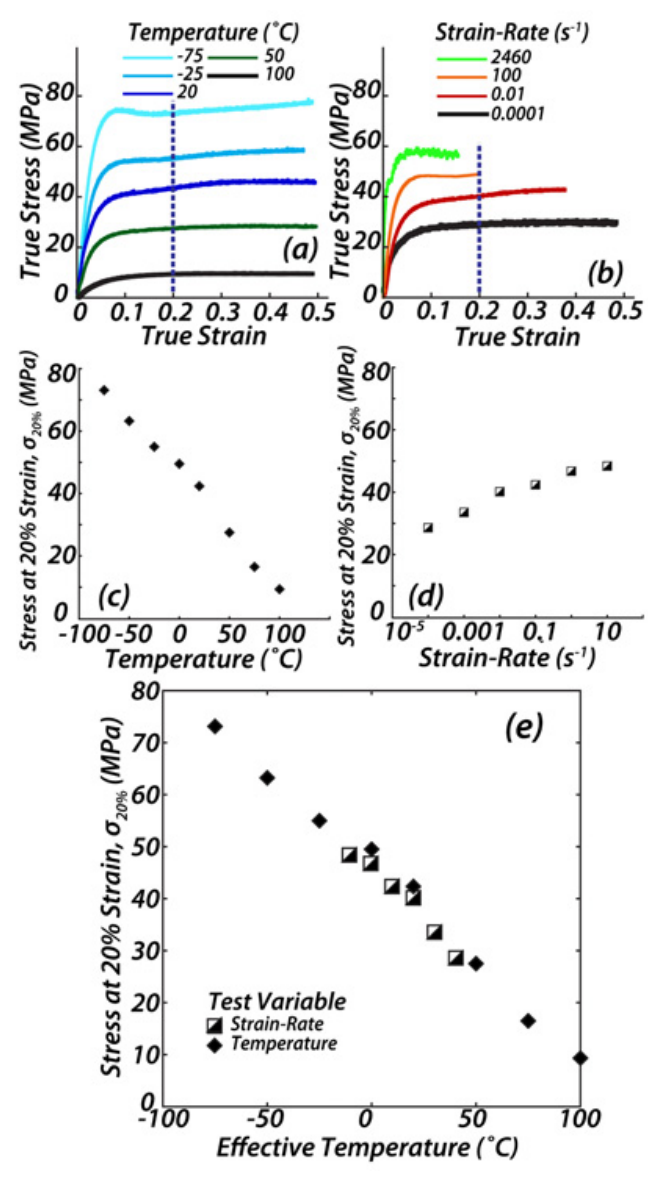

Fig. 2. (a) Stress-strain response of HDPE with varied temperature and (b) strain-rate. (c-d) Flow stress at 20\% strain from (a-b) respectively. (e) Eq. (1). applied to collapse (c-d) to a single equivalent response locus. After [7].

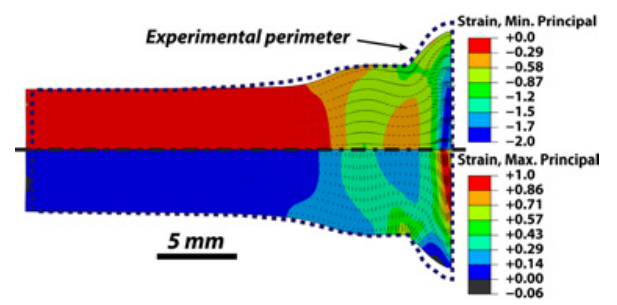

Fig. 3. FEA and experimental results for Taylor Impact of HDPE, shown at maximum compressive condition.

deformation occurs at strain-rates exceeding $10^{5} \mathrm{~s}^{-1}$ near the impact face, driven by the momentum of the trailing part of the projected specimen, much of which may not plastically deform at all (figure 3, 4(a)).

For a polymeric specimen, the material in contact with the anvil flows to large strains $[10,11,18,19]$, though in some cases for velocities above a ductile-to-brittle transition polymers may crack radially in an apparently brittle manner $[10,18]$. Initially, Taylor proposed to determine the dynamic yield stress by measuring the post impact total reduction of length and length of the plastically deformed region, but over the last few decades, the heterogeneous deformation that occurs during Taylor cylinder impact experiments have proven invaluable to verify more sophisticated constitutive models of the material response under extreme 


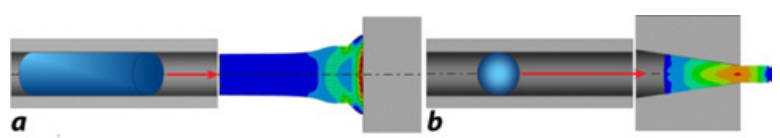

Fig. 4. (a) Schematic of Taylor impact and (b) Dynamic-TensileExtrusion.

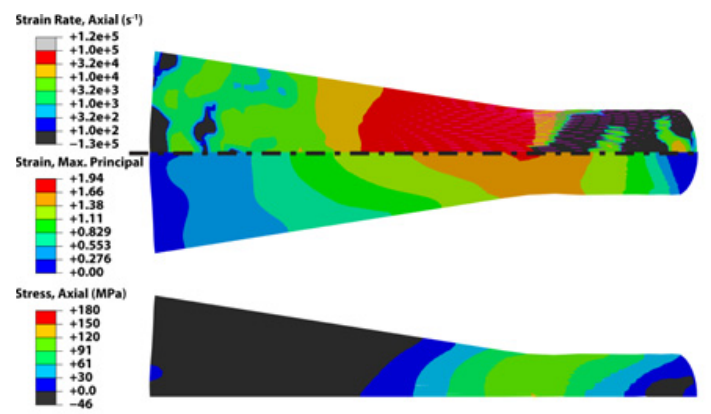

Fig. 5. FEA results of Dyn-Ten-Ext of HDPE at an initial velocity of $447 \mathrm{~m} \mathrm{~s}^{-1}$ and exit diameter $2.8 \mathrm{~mm}$.

compressive conditions [20]. As such, Taylor cylinder impact experiments provide valuable information about the dynamic inelastic response of a material, but do not probe principally tensile or shear-dominated states (i.e., the deformation occurs under hydrostatic compression). As many damage and failure phenomena are inhibited by pressure, a tensile analog to the Taylor cylinder impact technique enables the study the progression of such failure modes under extreme conditions.

The Dynamic-Tensile-Extrusion (Dyn-Ten-Ext) test was developed by Gray and co-workers at LANL to examine extreme tensile conditions in materials [21-23]. The apparatus utilizes the same hardware as a Taylor cylinder impact test, except that a conical extrusion die is fixed to the end of the gun barrel, forcing the specimen to extrude through it at a high velocity and the anvil is not employed. The leading edge of the specimen is relatively unaffected by the extrusion process, but the trailing portion rapidly decelerates inside the die, and thus the extruded ligament between the two ends is pulled in high strain-rate tension, typically to large strains and, ultimately, to failure (figure 4(b)).

As shown in figure 5, a finite element simulation ${ }^{\mathrm{a}}$ of high-density polyethylene (HDPE) undergoing Dyn-TenExt, through a die of exit diameter $3.60 \mathrm{~mm}$ with an initial velocity of $447 \mathrm{~s}^{-1}$, predicts that the ligament achieves a strain of 1 to 1.9 at a strain-rate of $10^{4}$ to $10^{5} \mathrm{~s}^{-1}$ in the critical tensile section during extrusion, thus achieving extreme tensile test conditions. Thus, as an integrated test that generates extreme tensile deformation, Dyn-Ten-Ext provides a means to challenge the quantitative accuracy of constitutive models parameterized under less extreme conditions. Additionally, it provides a direct qualitative assessment of the mechanisms of deformation and damage

\footnotetext{
${ }^{a}$ Exemplary FEA presented in the current work employed ABAQUS/Explicit using J2 plasticity with isotropic hardening and adiabatic heating, piecewise fit to HDPE quasi-static and SHPB stress-strain curves at various temperatures from [7] and validated against HDPE Taylor Impact images from [19], as shown in figure 3.
}

under extreme conditions, which may or may not differ from those observed at lower strain-rates.

\section{Materials and methods}

\subsection{Compression testing}

Compression tests were conducted with cylindrical specimens $6.2 \mathrm{~mm}$ in diameter and height on an MTS 880 servohydraulic load frame (MTS, Eden Prairie MN). The test specimens were machined from the same original stock of HDPE employed in previous work by the authors [7]. All specimens were measured with a micrometer prior to testing. The tests were conducted in strain control at a computed true strain-rate, and the strain was measured by an extensometer during the test mounted directly on the compression stage. Load, displacement, and strain were recorded both at regular time increments and at displacement increments of $1 \mu \mathrm{m}$.

The test temperature was controlled through compression platens cooled by nitrogen exhaust from a liquid nitrogen filled Dewar, and the temperature was controlled within $+/-0.5^{\circ} \mathrm{C}$ of the target temperature through manual adjustment of the gas flow rate. The specimens were loaded between optically flat tungsten carbide platens and were unlubricated. The lateral surfaces of the specimen were insulated from the environment with a foam sleeve. Temperature equilibrium was achieved by delaying testing until the displacement rate under a constant preload of 50 $\mathrm{N}$ was negligible $(<1 \mu \mathrm{m} / \mathrm{min})$. Two sets of jump rate tests were performed at $20^{\circ} \mathrm{C}$ and $-50^{\circ} \mathrm{C}$ up to a true strain of $100 \%$ in order to assess higher rate isothermal conditions. In each case the test was initiated at $d \varepsilon / d t=0.01 \mathrm{~s}^{-1}$, and either loaded at $d \varepsilon / d t=0.01 \mathrm{~s}^{-1}$ to completion or jumped to $d \varepsilon / d t=1 \mathrm{~s}^{-1}$ at strains of $10 \%, 20 \%, 30 \%$, and $40 \%$ (figure 6). Based on strain-rate-temperature superposition, baseline isothermal quasi-static tests where performed at temperatures depressed by $20^{\circ} \mathrm{C}$ and $30^{\circ} \mathrm{C}$ at strain-rates of $0.01 \mathrm{~s}^{-1}$ and $0.001 \mathrm{~s}^{-1}$, respectively.

\subsection{Dynamic-Tensile-Extrusion}

The Dyn-Ten-Ext apparatus employs a helium driven gas gun with a pneumatically actuated breech that can be pressurized to $4600 \mathrm{psi}$ and released remotely. The exit velocity of the projectile is controlled by choice of breach pressure, with velocities from 100 to $600 \mathrm{~ms}^{-1}$ easily achieved. The barrel has a smooth $7.62 \mathrm{~mm}$ (0.300 inches) bore. The barrel discharges into a target chamber that is evacuated with a roughing pump for testing to prevent interaction of the projectile with gas. While the chamber contains a polished hardened steel anvil during Taylor cylinder impact testing, for Dyn-Ten-Ext this is replaced with a catch tank loosely filled with cloth for soft recovery of any specimen fragments.

The main data collected during Dyn-Ten-Ext testing consist of initial velocity from the bore prior to interaction with the die and high speed multiframe photography of the extruded material for obtaining deformed shape and 
speed, which is then compared to FEA simulations to understand the material behavior during the test. The specimen initial velocity is measured via two pressure transducers on the barrel near the target chamber and captured with an oscilloscope. The images of the extruded material are captured with an Imacon 200 framing camera (Hadland, Santa Cruz CA), typically with a 50 ns shutter time and $3-5 \mu$ s interframe time, while illumination is provided opposite the camera by a Powerlight 2599 DR flash lamp (Photogenic, Bartlett IL). The flash lamp and camera are both activated after a programmed delay by a signal from an accelerometer attached to the die that triggers off of the sensed impact of the projectile with the die. The velocity of extruded material is calibrated with a transparent scale near the die exit.

The Dyn-Ten-Ext specimen geometry can be a sphere or a hemisphere-ended cylinder, both of which use a diameter and length of $7.60 \mathrm{~mm}$. The die uses a conical reducing section inclined 9 degrees (half-angle) to the extrusion axis. High hardness tool steel has been used in with metallic specimens, but stock stainless steel has been successfully employed for polymers. The die exit diameter is specified depending on the desired draw ratio to be imposed prior to the onset of dynamic self-extrusion after the die exit. The nominal design of the die uses an exit diameter of $2.80 \mathrm{~mm}$, which imposes an axial true strain $\varepsilon=2$ at the die exit.

\section{Results}

\subsection{Time (strain-rate)-temperature equivalence}

Using the temperature-strain-rate equivalence relation in Eq. (1) and $A=10.2^{\circ} \mathrm{C} /$ decade, quasi-static near-equivalent conditions HDPE can be directly compared to the results of the rate-jump tests by depressing the temperature by $20^{\circ} \mathrm{C}$ or $30^{\circ} \mathrm{C}$ for constant true strain-rates of $0.01 \mathrm{~s}^{-1}$ and $0.001 \mathrm{~s}^{-1}$, respectively (figure 6). A direct comparison of these quasi-static equivalent states and the rate-jump loci shows generally good agreement for strains exceeding $10 \%$.

At $20 \mathrm{C}$, the deformation response in HDPE showed a limited amount of softening at a strain-rate of $1 \mathrm{~s}^{-1}$; the flow stress was depressed by 3-5 MPa between $20 \%$ and $50 \%$ strain (figure 6(a)). The agreement with the predicted equivalent quasi-static tests was good.

The response at $-50 \mathrm{C}$ in HDPE exhibits considerable softening, with the flow stress dropping approximately $10 \mathrm{MPa}$ after each rate jump (figure 6(b)). The $1 \mathrm{~s}^{1}$ response softens to the point that the flow stress is lower at large strains than that at $0.01 \mathrm{~s}^{-1}$ - a hallmark of adiabatic heating effects. With such severe specimen heating, a rate-jump approach is necessary to form isothermal stress-strain curves.

\subsection{Dynamic-Tensile-Extrusion}

Dyn-Ten-Ext tests in general yield two types of observable behavior: stable continuum deformation of an extruded

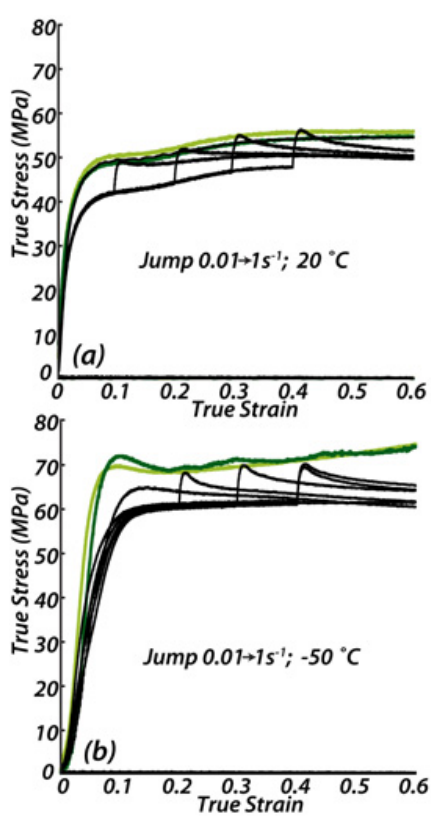

Fig. 6. Deformation response for HDPE for a jump condition (black) of $0.01->1 \mathrm{~s}^{-1}$ strain-rate at (a) $20 \mathrm{C}$ and (b) $-50 \mathrm{C}$. Equivalent states (green) at 0.001 and $0.01 \mathrm{~s}^{-1}$ agree with the isothermal jump locus.

jet, then potentially followed by terminal behavior, e.g., damage, failure, or fragmentation. The former is studied to verify continuum descriptions of material behavior and develop continuum failure criteria, while the latter can be used to investigate dynamic instabilities and the final stages of damage progression. At the interface between stable deformation and failure is the progression of damage beyond the incipient condition, which may follow a complicated path under the simultaneous influence of multiple driving forces. It should also be noted that some test conditions will yield only deformation with no damage for a particular material, while under other conditions that material might fail soon after or even prior to extruding and thus yield little information about continuum behavior.

\subsubsection{Continuum deformation}

Extreme tensile continuum deformation of polymers is readily observed in Dyn-Ten-Ext, either as the terminal state in more moderate tests or up to the point of incipient failure. Even the case of a large die draw ratio of 7.5, HDPE extrudes a long jet prior to the onset of local instability and failure (figure 7(a)).

The extruded jet is slightly larger in diameter than the die exit, and much of it experiences only a small increment of strain beyond that imposed by extrusion after passing out of the critical tensile section (i.e., vanishing strain-rate beyond the die; figure 5).

Depending on the conditions of the experiment (e.g., draw ratio and initial velocity) the critical tensile section (tensile strain-rate exceeding $10^{4} \mathrm{~s}^{-1}$ ) may occur within the die, limiting the interpretability of the test via external observation of extrusion. Finite element analysis must therefore be used to predict favorable test conditions for 


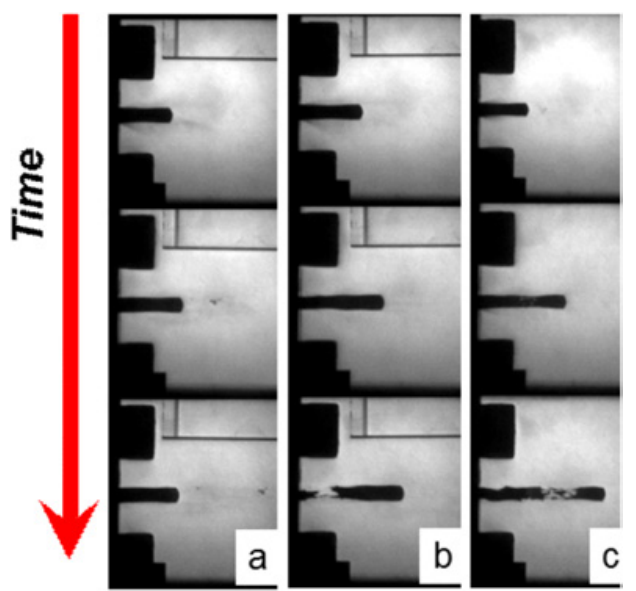

Fig. 7. Dynamic-Tensile-Extrusion at (a) low, (b) intermediate, and (c) high velocity. Damage is (a) stable and limited, (b) stable and terminal, and (c) unstable and catastrophic.

ensuring that the material behavior of interest can be observed during the experiment. It is important to note that during extrusion, while the specimen is being drawn in the die, it is under high hydrostatic pressure and potentially severe frictional loading, and these parts of the test are challenging to observe directly. Notwithstanding this limitation, it is assumed that the influence of these two factors imposes a negligible influence on the subsequent extreme tensile phase of the test. However, similar pressures and frictional loading occur in the Taylor cylinder impact test, where they are observable and therefore can inform the appropriateness of the model conditions during extrusion. This further motivates Dyn-Ten-Ext and Taylor cylinder impact as complimentary extreme mechanical tests.

\subsection{Damage and Terminal Behavior}

In most cases where extrusion takes place the jet breaks down in the terminal deformation stage either in a localization failure or fragmentation in a more diffuse zone of failure (figure 7(a)-(c)). A range of results is obtained as the input velocity is increased, progressing from limited damage to terminal damage progression, to catastrophic failure by a separate mechanism than seen at lower velocities. This is a useful set of results, because it captures the evolution of damage under extreme tensile conditions to failure, and also reveals that two separate mechanisms (or at least pathways) of damage are competing during the deformation.

The progression of damage to failure is evident upon cross-section of the recovered specimens (figure 8). It is clear that damage progresses along a pseudo-axial path that links an internal flaw to the exterior edge of the specimen (figure 8(a)), where rupture occurs at higher velocities at the die exit. Closer examination of the intact specimen (figure 8(b)) reveals an internal failure at the specimen axis in the form of a macroscopic shear crack, which is followed by a shear-mode pull-out of the core behind the flaw in a strain concentrating process. X-ray tomography reveals the damage mechanism to be a shear-cracking process (figure $8(\mathrm{c})$ ).

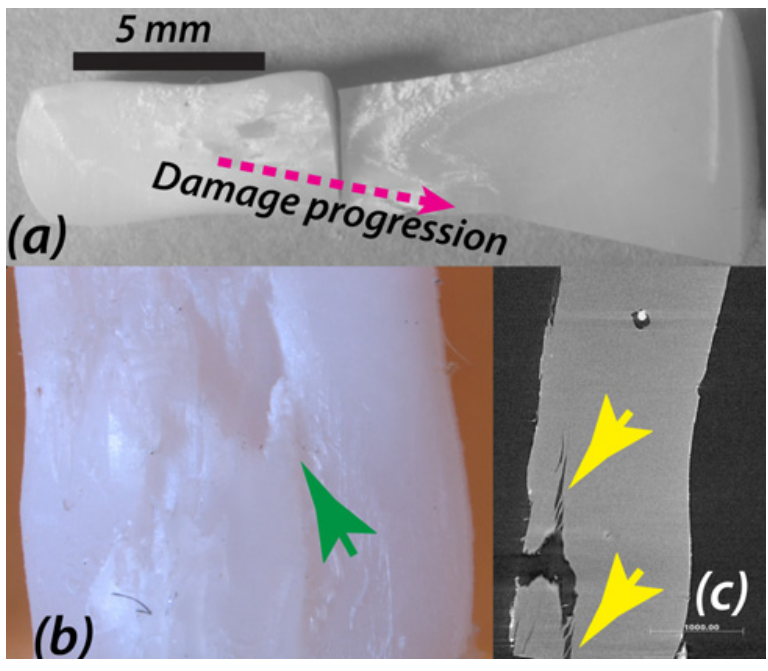

Fig. 8. Postmortem of low-velocity HDPE Dyn-Ten-Ext with stable damage. (a) Cross-section of recovered specimen. (b) Section detail showing chevron tensile failure and shear pull-out damage. (c) X-ray computed tomography showing shear-mode cracks in damage region ahead of and behind chevron failure.

Both the shear-failure and apparent catastrophic crazelike behavior seen in Dyn-Ten-Ext of HDPE are processes strongly exacerbated by hydrostatic tension, which may explain why they undergo stable deformation past the die exit and not during large strain extrusion under pressure within the die.

High density polyethylene appears to undergo a pressure-dependent shear-damage mechanism, though the mechanism of damage may be somewhat distinct from crazing which has been observed in HDPE, while extrapolation of a constitutive model from lower strain-rate data to Dyn-Ten-Ext strain rates captured the deformation response very well. Further work may elucidate the exact nature of the failure in Dyn-Ten-Ext of these two polymers by altering the test conditions (for reduced pressure).

\section{Discussion and Conclusions}

The agreement of the isothermal deformation response at quasi-static and elevated strain-rates in HDPE and UHMWPE, over a wide range of plastic strains, extends the applicability of time-temperature equivalence techniques in these materials into the large strain regime. This significantly improves the efficacy of time-temperature equivalence relations by enabling the translation of continuous stress-strain curves obtained in the laboratory at quasi-static isothermal conditions into dynamic regimes of behavior at substantially elevated strain-rates. The ability to shift experimental stress-strain curves is extremely valuable in the development or evaluation of rate-dependent constitutive models, where model predictions of difficultto-test extreme mechanical conditions (e.g., large strains at high strain-rates) can be directly compared to timetemperature equivalent conditions from laboratory data. Time-temperature equivalence holding over a wide range of strains and temperatures also implies that one mechanism of temperature-dependence is active in general, 
which is also useful information for model development. It is also of note that the jump locus coincides directly with the equivalent state, apparently without substantial overshoot or undershoot. In metals, such a disagreement is linked to the pinning or accumulation of dislocations (respectively), making the rate-sensitivity a history dependent phenomenon. The rate-sensitivity in HDPE and UHMWPE, and hence their time-temperature equivalence, appear to be history-independent and therefore generally applicable.

The Dynamic-Tensile-Extrusion technique is a relatively simple and effective experimental approach for studying the high strain-rate and large strain constitutive behavior of materials, with a particular potential for ductile polymers that exhibit both high strain-to-failure and strong variation with strain-rate. As an integrated test, results from Dyn-Ten-Ext requires finite element analysis for the interpretation of stress and strain fields, which in turn requires a material model validated in the regime of strain-rate active under similar mechanical conditions. The temperature-strain-rate equivalence approach investigated in this work was used to adapt conventional stress-strain curves into the extreme deformation regime, and this approach was validated via prediction of a Taylor impact test. Thus, the synthesis of time-temperature equivalence, a battery of large-strain characterization tests, and Taylor impact studies present a validated approach for the investigation of polymers under extreme tensile conditions. Both deformation and failure phenomena under extreme mechanical conditions can be directly observed in DynTen-Ext, which in turn reveals the phenomena driving damage and failure in the material. As an integrated test with complicated stress, strain, and strain-rate gradients, with simple well-defined boundary conditions, Dyn-TenExt is well suited for the refinement and validation of material models under extreme conditions.

\section{Acknowledgements}

Los Alamos National Laboratory is operated by LANS, LLC, for the NNSA of the US Department of Energy under contract DE-AC52-06NA25396. This research was supported by Campaign 2: Dynamic Behavior of Materials and the Joint DoD/ DOE Munitions Program. The authors thank Carl Trujillo and Daniel Martinez for assistance with Dynamic-Tensile-Extrusion testing.

\section{References}

1. D.J. Funk, G.T.G. III, T.C. Germann, R.L. Martineau, Los Alamos National Laboratory Report LA-UR-0907771. (2009)

2. G.T. Gray III, W.R. Blumenthal, ASM Handbook Volume 8: Mechanical Testing and Evaluation. 8, 488 (2000)

3. P.J. Rae, D.M. Dattelbaum, Polymer. 45, 7615 (2004)

4. C.R. Siviour, S.M. Walley, W.G. Proud, J.E. Field, Polymer. 46, 12546 (2005)

5. J. Richeton, S. Ahzi, K.S. Vecchio, F.C. Jiang, et al., Int J Solids Struct. 43, 103 (2006)

6. C.R. Siviour, S.M. Walley, W.G. Proud, J.E. Field, J Phys IV. 134, 949 (2006)

7. E.N. Brown, R.B. Willms, G.T. Gray, P.J. Rae, et al., Exp Mech. 47, 381 (2007)

8. J.L. Jordan, C.R. Siviour, J.R. Foley, E.N. Brown, Polymer. 48, 4184 (2007)

9. E.N. Brown, P.J. Rae, G.T. Gray, J Phys IV. 134, 935 (2006)

10. E.N. Brown, P.J. Rae, E.B. Orler, Polymer. 47, 7506 (2006)

11. P.J. Rae, E.N. Brown, E.B. Orler, Polymer. 48, 598 (2007)

12. Rae, E.N. Brown, Polymer. 46, 8128 (2005)

13. H. Eyring, J Chem Phys. 3, 107 (1935)

14. H. Eyring, J Chem Phys. 4, 281 (1936)

15. M.L. Williams, R.F. Landel, J.D. Ferry, J Am Chem Soc. 77, 3701 (1955)

16. S. Hillmansen, R.N. Haward, Polymer. 42, 9301 (2001)

17. G. Taylor, Proc R Soc Lon Ser-A. 194, 289 (1948)

18. P.J. Rae, E.N. Brown, B.E. Clements, D.M. Dattelbaum, J Appl Phys. 98, 063521 (2005)

19. E.N. Brown, C.P. Trujillo, G.T. Gray, Shock Compression of Condensed Matter - 2007. AIP Conf Proc. 955, 691 (2007)

20. P.J. Maudlin, J.F. Bingert, G.T. Gray, Int J Plasticity. 19, 483 (2003)

21. F. Cao, E.K. Cerreta, C.P. Trujillo, G.T. Gray, Acta Mater. 56, 5804 (2008)

22. G.T. Gray, E. Cerreta, C.A. Yablinsky, L.B. Addessio, et al., Shock Compression of Condensed Matter 2005, AIP Conf Proc. 845, 725 (2006)

23. E.N. Brown, C.P. Trujillo, G.T. Gray III, Shock Compression of Condensed Matter - 2009, AIP Conf Proc. 1195, 1233 (2009) 